\title{
El control estadístico de la calidad en la técnica del hormigón
}

Se acostumbra evaluar según las leyes de la estadística matemática los resultados de investigaciones relacionadas entre sí y expresarlos mediante valores característicos que permitan clasificar determinadas propiedades de las muestras tomadas al azar. La conclusión que se obtiene se refiere a la totalidad y se consigue así una imagen del estado del material que hay que enjuiciar.

Por "totalidad básica" se entiende un conjunto de muestras equivalentes, en las que se van a examinar determinadas propiedades. Para este fin se representa el conjunto del objeto que hay que someter a examen, separado en individuos o clases, por ejemplo hormigón de núcleo u hormigón de un embalse o presa; para mencionar otro ejemplo, la producción mensual de un grupo de armaduras de hormigón. Si el número de los elementos representables en comparación con el volumen demasiado reducido de la muestra al azar es muy grande, se habla de un conjunto básico "infinito"; en otro caso, de conjunto básico "finito". Se llaman muestras al azar, por el contrario, a las cantidades parciales propiamente dichas, tomadas del conjunto básico, que se examinan y cuyos resultados se utilizan para una ulterior elaboración. Se examina, por tanto, un número relativamente reducido de muestras y los resultados hallados se aplican al conjunto del objeto que hay que examinar: al hormigón mencionado o, en el caso del segundo ejemplo, a todo el montón de varillas de armadura. Este tipo de proceso, sin embargo, solamente es conveniente si la toma de muestras al azar representa una selección casual, en la que tienen oportunidad de ser tomadas todas las muestras posibles; además ninguna muestra puede estar afectada de un defecto sistemático. Por consiguiente, en una preparación del hormigón que se ha de emplear debe resultar tan buena una mezcla como cualquier otra y preparada en las mismas condiciones. En especial los valores teóricos del contenido de cemento, del valor a/c, la granulometría, etc., deben ser siempre los mismos. Unicamente bajo estas condiciones puede hacerse responsable a la casualidad, que está fuera de cálculo, de las inevitables dispersiones de los resultados.

En la elaboración estadística de los resultados de ensayo lo mejor es proceder según DIN 55302 , hoja 1. En este caso principalmente interesa la desviación estándar $s$ y el coeficiente de variación $v$.

La desviación estándar $s$ aumenta en muchos tipos de hormigón con el desarrollo de las resistencias; por ello en cualquier hormigón, especialmente en el hormigón en masa, que la mayor parte de las veces se examina con una edad superior a 28 días, debe indicarse expresamente la fecha del ensayo. Como ejemplo pueden servir las desviaciones estándar de un hormigón con $170 \mathrm{~kg} / \mathrm{m}^{3}$ de cemento y otro con $240 \mathrm{~kg} / \mathrm{m}^{3}$ de cemento contenidos en la tabla 1. Los demás valores característicos son los mismos en ambas mezclas. Hay que resaltar que aquí se trata de un cemento con un marcado fraguado lento, un árido de mediana calidad y de un tamaño máximo de $120 \mathrm{~mm}$. 
TABLA 1

Desviación específica $s\left(\mathrm{kp} / \mathrm{cm}^{2}\right)$

\begin{tabular}{|c|c|c|}
\hline $\begin{array}{c}\text { Edad } \\
\text { (días) }\end{array}$ & Hormigón de $170 \mathrm{~kg} / \mathrm{m}^{3}$ & Hormigón de $240 \mathrm{~kg} / \mathrm{m}^{3}$ \\
\cline { 2 - 3 } & \multicolumn{3}{|c|}{ Resistencias a compresión } \\
\hline 7 & 15 & 25 \\
90 & 35 & 30 \\
180 & 35 & 35 \\
\hline
\end{tabular}

Los valores $s$ aumentan en este caso hasta los 90 días y a continuación permanecen invariables hasta los 180 días.

La importancia de la desviación estándar como medida para la calidad alcanzada en la preparación del hormigón y el cuidado puesto en ello, se expresa claramente en las dos tablas siguientes.

La tabla 2 informa, en virtud del valor medio de la resistencia a la presión y de la desviación estándar calculada, acerca de la uniformidad de la preparación del hormigón.

TABLA 2

Calificación del hormigón con ayuda de la desviación específica o estándar s

\begin{tabular}{|c|c|c|}
\hline $\begin{array}{l}\text { Resistencia media a la compresión } \\
\qquad\left(\mathbf{k p} / \mathbf{c m}^{2}\right)\end{array}$ & \multicolumn{2}{|c|}{$\left.\right|_{\text {buena }} ^{\text {Uniformidad en la preparación del hormigón }}$ mala } \\
\hline 200 & $\leqslant 45$ & $\geqslant 60$ \\
\hline 300 & $\leqslant 49$ & $\geqslant 64$ \\
\hline 400 & $\leqslant 53$ & $\geqslant 68$ \\
\hline 500 & $\leqslant 57$ & $\geqslant 72$ \\
\hline 600 & $\leqslant 60$ & $\geqslant 75$ \\
\hline
\end{tabular}

El contenido de esta tabla tiene un valor meramente informativo y por ello sólo debe aplicarse como directriz, porque el criterio para el enjuiciamiento de la calidad del hormigón se rige por las circunstancias que dominan las diferentes obras, en las que se prepara el hormigón. Esto mismo se puede deducir de la siguiente tabla 3, en la que las desviaciones estándar relacionadas con una determinada magnitud de las obras y una influencia de la intemperie permiten deducir el cuidado empleado en la fabricación del hormigón.

El coeficiente de variación

$$
v=\frac{s \cdot 100}{\bar{x}} \%
$$

contiene, además de la desviación estándar $s$, el valor medio $\bar{x}$, y por ello es especialmente expresiva para el enjuiciamiento de la uniformidad de un hormigón fabricado a lo largo de un período prolongado de tiempo con una relación invariable de mezcla. Para el cálculo de diferentes clases de hormigón, con notables diferencias de resistencia por debajo 
de los valores medios $\bar{x}$, no es sin embargo tan adecuada como la desviación estándar; por ejemplo en dos mezclas, con aproximadamente la misma dispersión o desviación estandar $s$, se ajustarían dos diferentes coefientes de variación de acuerdo con la diferencia existente entre $\bar{x}_{1}$ y $\bar{x}_{2}$, como lo muestra el siguiente ejemplo (tabla 4).

T A в L A 3

Apreciación del cuidado empleado en la preparación del hormigón, con ayuda de la desviación específica $s$ (3)

\begin{tabular}{|c|c|c|c|c|}
\hline \multirow{3}{*}{$\begin{array}{l}\text { Cuidado empleado en la } \\
\text { preparación del hormigón }\end{array}$} & \multicolumn{4}{|c|}{ Desviación específica $\left(\mathbf{k p} / \mathrm{cm}^{2}\right)$} \\
\hline & \multicolumn{4}{|c|}{ Influencia de la intemperie } \\
\hline & Grande* & Pequeña* & Grande $* *$ & Pequeña $* *$ \\
\hline Muy bueno & 50 & 40 & 40 & 30 \\
\hline Bueno & 60 & 50 & 50 & 40 \\
\hline Aceptable & 70 & 60 & 60 & 50 \\
\hline
\end{tabular}

* Dosificación según partes de volumen; control por el aparejador.

** Dosificación según partes de peso; control por parte del ingeniero de hormigón.

T A B L A 4

\begin{tabular}{|c|c|c|}
\hline & \multicolumn{2}{|c|}{ Gran embalse } \\
\hline & $\begin{array}{c}\text { Hormigón del } \\
\text { núcleo }\end{array}$ & $\begin{array}{c}\text { Hormigón de la } \\
\text { superficie }\end{array}$ \\
\hline $\begin{array}{llllllll}\text { Dosificación de cemento } & \ldots & \ldots & \ldots & \ldots & \ldots & \ldots & \left(\mathrm{kg} / \mathrm{m}^{3}\right)\end{array}$ & 165 & 240 \\
\hline $\begin{array}{llllllllll}\text { Número de muestras } & \ldots & \ldots & \ldots & \ldots & \ldots & \ldots & \ldots & \ldots & \ldots\end{array}$ & 195 & 199 \\
\hline Edad del ensayo $\ldots \begin{array}{lllllllll} & \ldots & \ldots & \ldots & \ldots & \ldots & \ldots & \ldots & \ldots \\
\text { (días) }\end{array}$ & 180 & 180 \\
\hline Valor medio de la resistencia a compresión $\left(\mathrm{kp} / \mathrm{cm}^{2}\right)$ & 217 & 326 \\
\hline 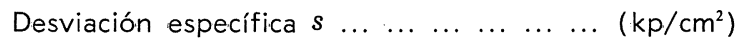 & 33 & 34 \\
\hline Coeficiente de variación $\begin{array}{lllllll}v & \ldots & \ldots & \ldots & \ldots & \ldots & \left(\mathrm{kp} / \mathrm{cm}^{2}\right)\end{array}$ & 15 & 10 \\
\hline
\end{tabular}

Se acostumbra a calificar la preparación del hormigón, en virtud del coeficiente de variación $v$, como sigue:

\begin{tabular}{|l|c|c|c|c|}
\hline Coficiente de variación v, en $\%$ & 10 & 15 & 20 & 25 \\
\hline Calificación & Muy bien & Bien & Aceptable & Mal \\
\hline
\end{tabular}

Es digna de conocerse la tabla 5, que procede de los Estados Unidos, con los coeficientes de variación decisivos respecto de la calidad de hormigón de obra y de laboratorio. Los valores límite de estos coeficientes recopilados en grupos tienen asimismo sólo un carácter informativo.

La desviación total $s$ se compone de la desviación de mezcla $s_{\mathrm{m}}$ y de la desviación de probetas $s_{\mathrm{p}}$ que se relacionan según la fórmula de Pitágoras $s^{2}=s_{\mathrm{m}}^{2}+s_{\mathrm{p}}^{2}$. 
T A B L A 5

Calificación de la preparación del hormigón con auxilio del coeficiente de variación (4)

\begin{tabular}{|c|c|c|c|c|}
\hline \multirow{2}{*}{ Valor de la dispersión } & \multicolumn{4}{|c|}{$\begin{array}{c}\text { Coeficiente de variación, como medida del cuidado aplicado a la preparación } \\
\text { del hormigón }(\%)\end{array}$} \\
\hline & Muy bueno & Bueno & Suficiente & Malo \\
\hline \multicolumn{5}{|l|}{ Dispersión total $\mathrm{s}$} \\
\hline En obra & $<10$ & 10 a 15 & 15 a 20 & $>20$ \\
\hline En el laboratorio & $<5$ & 5 a 7 & 7 a 10 & $>10$ \\
\hline \multicolumn{5}{|l|}{ Dispersión de productos $p$} \\
\hline En obra & $<4$ & 4 a 5 & 5 a 6 & $>6$ \\
\hline En el laboratorio & $<3$ & 3 a 4 & 4 a 6. & $>5$ \\
\hline
\end{tabular}

Por desviación de mezcla $s_{\mathrm{m}}$ se entiende la desviación estándar de las resistencias medias de probetas de cada una de las cargas de la hormigonera $\bar{x}_{\mathrm{i}}$ en relación con la media común de todas las probetas $\bar{x}$ o, en una palabra: la desviación entre las mezclas; mientras que la desviación de probetas $s_{\mathrm{p}}$ significa la desviación estándar de las resistencias de todas las probetas con los valores medios de las mezclas correspondientes a la desviación estadística de una:

$$
s_{\mathrm{m}}=\sqrt{\frac{\sum\left(\bar{x}_{\mathrm{i}}-\bar{x}\right)^{2}}{n-1}} \quad ; \quad \bar{x}=\frac{\sum \bar{x}_{\mathrm{i}}}{n}
$$

$i(1,2, \ldots n) \ldots . .$. mezclas

$$
s_{\mathrm{p}}=\sqrt{\frac{\sum\left(x_{\mathrm{ik}}-\bar{x}_{\mathrm{i}}\right)^{2}}{n-1}}
$$

$i(1,2 \ldots r) \ldots \ldots$ mezclas

$k(1,2 \ldots \alpha ; 1,2 \ldots \beta ; 1,2 \ldots \omega) \ldots \ldots$ probetas

$n(\alpha+\beta+\ldots+\omega) \ldots . .$. número total de probetas.

La magnitud de la desviación de probetas $s_{\mathrm{p}}$ es, como han demostrado los análisis, considerablemente menor que la magnitud de la desviación de calidad $s_{\mathrm{m}}$ : sólo alcanza a un $20 \%$ de la desviación total.

Las dos magnitudes de desviación $s_{\mathrm{m}}$ y $s_{\mathrm{p}}$ están dominadas principalmente por las siguientes influencias:

Desviación de la mezcla $s_{\mathrm{m}}$ :

a) Variación del valor a/c (determinación insuficiente de la humedad propia del material árido que oscila la mayor parte de las veces dentro de unos amplios límites; inexacta adición de agua).

b) Variación de la demanda de agua (variación de la granulometría de los áridos).

c) Variación de las propiedades y de las cantidades de componentes (cemento, áridos, aditivos, agentes).

d) Irregularidades en el transporte, colocación y compactación del hormigón.

e) Diferente tratamiento posterior de las muestras. 
Desviación de probetas $s_{\mathrm{p}}$ :

a) Toma inadecuada de muestras y condiciones de fabricación dudosas (insuficiente tiempo de mezcla, diferente granulación máxima, compactación irregular).

b) Diferente tratamiento posterior de cada una de las muestras (temperatura variante y humedad).

c) Malas condiciones del ensayo (superficies de presión desiguales, probetas inexactas, diferente velocidad de carga, probeta inadecuadamente centrada en la prensa de ensayo).

De la costumbre habitual, de tomar de una mezcla tres probetas y ensayarlas, se ha pasado ahora al convencimiento de que la desviación de probetas sólo ejerce una reducida influencia sobre la desviación total. Por tanto en la práctica no interesa tanto la desviación de probetas, como la desviación de la mezcla. Por ello es más conveniente distribuir casi regularmente el número de probetas tomadas a lo largo de todo el día; tomar una probeta de cada mezcla entresacada, con la que poder adquirir una información más profunda de las circunstancias que causan la desviación del hormigón.

El coeficiente de variación, cuya fecha de ensayo debe indicarse siempre, se podrá aplicar con buen resultado solamente si una determinada calidad de hormigón se mantiene en el programa sin variación alguna a lo largo de un período prolongado de tiempo. Es conveniente citar asimismo la correspondiente desviación estándar. $\mathrm{Si}$, por el contrario, existe la necesidad de comparar entre sí la calidad de los hormigones producidos en diferentes obras, la mejor medida para esto es la desviación estándar. No en vano se le ha conferido la denominación de "constante de cuidado". En la figura 1 se han representado las características de la curva de distribución de Gauss o Normal y sus relaciones.
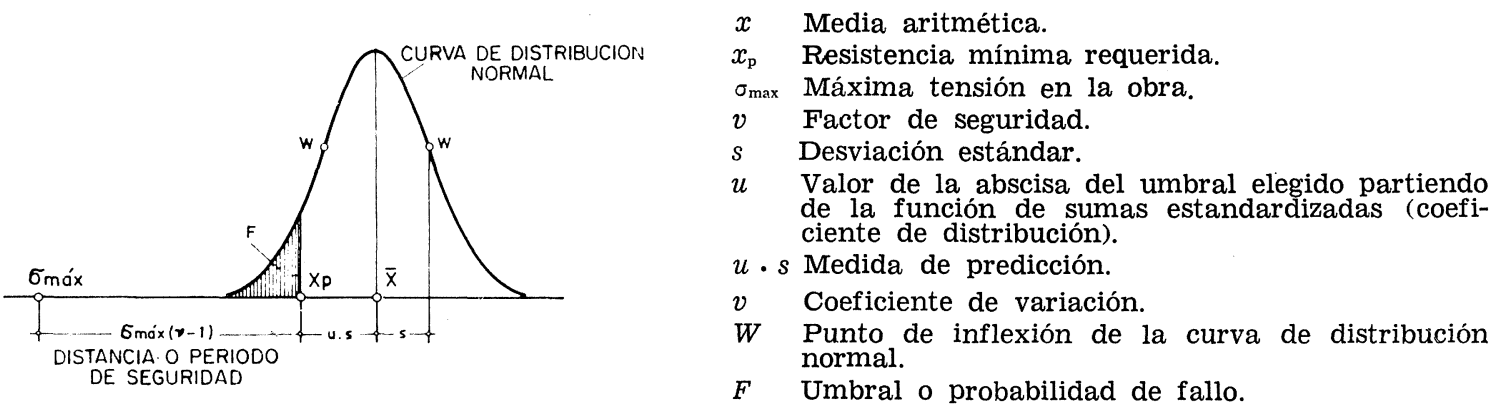

Fig. 1

Como umbral de probabilidad del resultado se designa a la parte del número total de muestras, cuyos resultados son menores que la resistencia mínima requerida. Según las condiciones que el cliente está dispuesto a fijar teniendo en cuenta la clase e importancia de la obra para la empresa constructora, la probabilidad varía entre 5 y $15 \%$. En obras de hormigón armado expuestas a grandes esfuerzos se tomará generalmente la probabilidad de $5 \%$; en el caso de muros de presa, el $10 \%$, y en obras menos sensibles, el $15 \%$.

Un ejemplo puede explicar más detalladamente la significación en la figura 1: la resistencia por compresión decisiva alcanza en una obra $60 \mathrm{kp} / \mathrm{cm}^{2}$ y el factor de seguridad 4 . De aquí se deduce que la resistencia mínima requerida es $x_{\mathrm{p}}=4 \times 60=240 \mathrm{kp} / \mathrm{cm}^{2}$. Se elegiría la probabilidad del $10 \%$, teniendo en cuenta la instalación de la obra y el personal disponible, para que puedan alcanzarse coeficientes de variación $v=10 \%$. El coeficiente de distribución $u$ se encuentra en la tabla 6. Para una probabilidad del $10 \%$ es 1,28 (tabla 6). 
TABLA 6

Coeficientes de variación u para algunos márgenes de probabilidad

\begin{tabular}{|cc|cc|}
\hline márgenes de probabilidad \% & $\mathbf{u}$ & márgenes de probabilidad \% & $\mathbf{u}$ \\
\hline 1 & 2,3264 & 11 & 1,2266 \\
2 & 2,0538 & 12 & 1,1750 \\
3 & 1,8808 & 13 & 1,1264 \\
4 & 1,7507 & 14 & 1,0803 \\
5 & 1,6449 & 15 & 1,0364 \\
6 & 1,5548 & 16 & 0,9945 \\
7 & 1,4758 & 17 & 0,9542 \\
8 & 1,4051 & 18 & 0,9154 \\
9 & 1,3408 & 19 & 0,8779 \\
10 & 1,2815 & 20 & 0,8416 \\
\hline
\end{tabular}

De aquí resulta:

$$
\bar{x}=\frac{x_{\mathrm{p}}}{1-v \cdot u}=\frac{240}{1-0,10 \cdot 1,28}=275 \mathrm{kp} / \mathrm{cm}^{2}
$$

Con un número mayor de ensayos de calidad -más 25 ó $30-$ el valor medio de las resistencias a compresión, según esto, se encuentra en $275 \mathrm{kp} / \mathrm{cm}^{2}$. Por tanto en la comprobación de idoneidad se debe buscar la mezcla cuya resistencia media a compresión, en la fecha convenida, alcance por lo menos $275 \mathrm{kp} / \mathrm{cm}^{2}$ y de este modo quedará a un $15 \%$ por encima del valor mínimo requerido $x_{\mathrm{p}}$.

La tabla 7 contiene las resistencias medias a compresión $\bar{x}$ correspondientes a resistencias mínimas comprendidas entre 120 y $300 \mathrm{kp} / \mathrm{cm}^{2}$, y umbrales de 5,10 y $15 \%$ y los coeficientes de variación $5,10,15,20$ y $25 \%$ según la fórmula arriba indicada. En esta tabla todos los valores medios, que corresponden al umbral de $10 \%$ y coeficiente de variación del $10 \%$, se encuentran aumentados en un $15 \%$ por encima de la resistencia mínima requerida, como lo exigía la antigua DIN 1 048/1943, 1a, en virtud de similares consideraciones.

En la nueva norma DIN 1 045/1972 en 7.4.2.2. se exige que las resistencias medias a compresión, que se alcancen durante el ensayo de idoneidad, superen ciertos valores de la tabla en determinados valores experimentales. La ONORM B 4200 (parte 10, 1971) prescribe en el número 5.2.2. que las resistencias en el ensayo de idoneidad deben superar un mínimo de $30 \mathrm{kp} / \mathrm{cm}^{2}$ a las de la tabla 2 .

Para un número menor de muestras, por ejemplo 25 ó 30 , se aplica la distribución - $t$, publicada por el irlandés U. S. Gosset el año 1908, que se conoce también con el nombre de distribución "Student", porque el estadístico Gosset ha firmado siempre sus trabajos científicos con este seudónimo. Esta distribución resulta cada vez más similar a la distribución normal de Gauss o a la curva de campana; con un número creciente de muestras, y un número superior a 30 no se puede percibir ya diferencia alguna considerable. Si la cantidad de muestras $n$ o el número de grados de libertad $m=n-1$ es infinitamente grande, entonces la función de la densidad de la distribución Student se convierte en la distribución normal. 
La función de densidad de la distribución Student para el grado de libertad $m$ es:

$$
g_{\mathrm{m}}(t)=\frac{1}{\sqrt{m \cdot \pi}} \cdot \frac{\Gamma\left(\frac{m+1}{2}\right)}{\Gamma\left(\frac{m}{2}\right)} \cdot \frac{1}{\left(1+\frac{t^{2}}{m}\right)^{\frac{m+1}{2}}}
$$

TAB L A 7

Valores medios $\bar{x}$ de la resistencia a la compresión calculados a partir del valor medio $x_{\mathrm{p}}$ deducido del coeficiente de variación $v$ y del margen de probabilidad

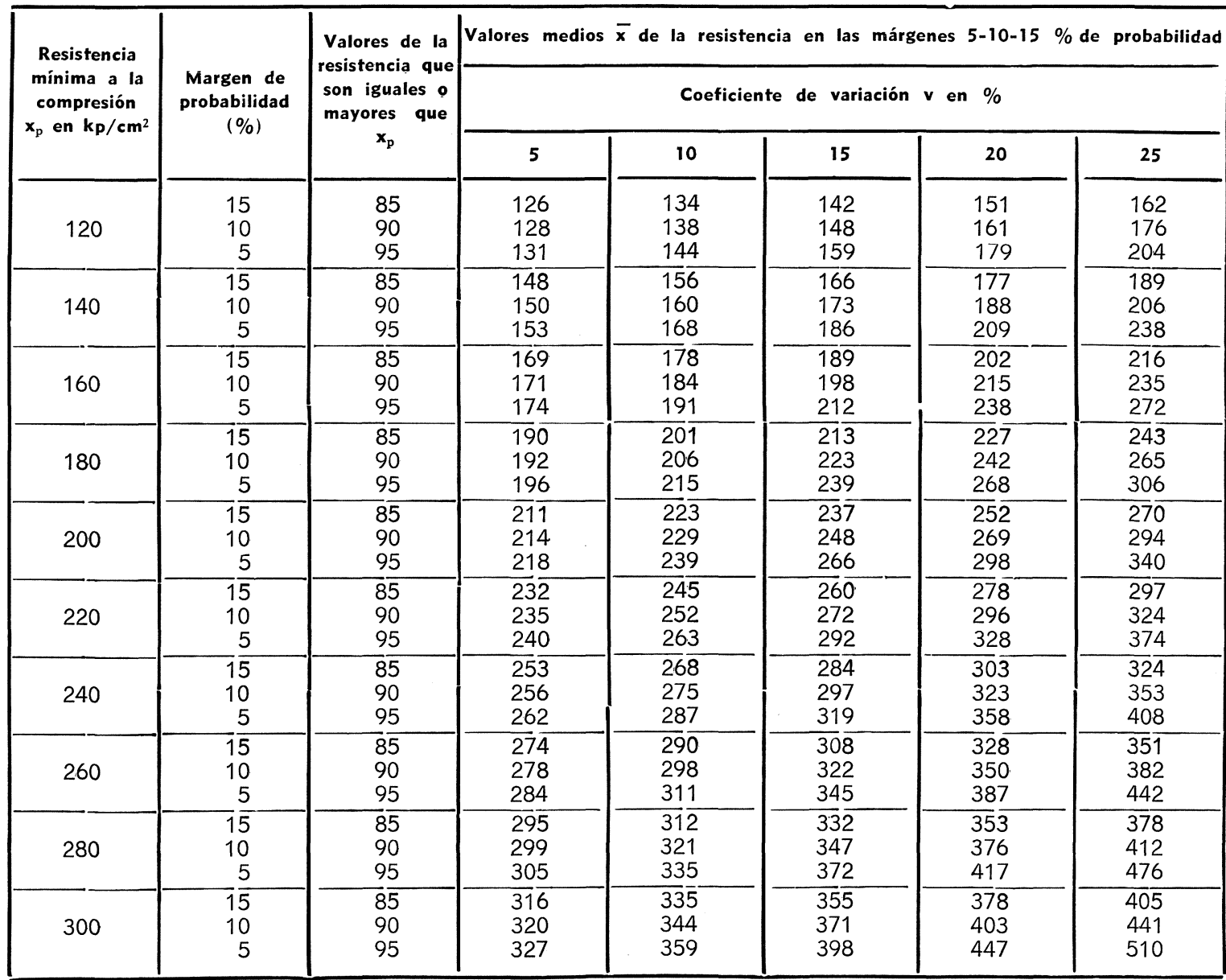

$$
\bar{x}=\frac{x_{\mathrm{p}}}{1-v \cdot u}
$$

$u=$ coeficiente de probabilidad.

$v=$ coeficiente de variación.

Para $-\infty<t<+\infty$.

$$
\begin{gathered}
\lim _{m \rightarrow \infty}\left(1+\frac{t^{2}}{m}\right)^{\frac{m+1}{2}}=\lim _{m \rightarrow \infty}\left(1+\frac{t^{2} / 2}{m / 2}\right)^{m / 2} \cdot \lim _{m \rightarrow \infty}\left(1+\frac{t / 2}{m / 2}\right)^{1 / 2}= \\
=\lim _{m \rightarrow \infty}\left(1+\frac{t^{2} / 2}{m / 2}\right)^{m / 2} \cdot 1=e^{t 2 / 2}
\end{gathered}
$$


Según la fórmula de Stirlingschen, se obtiene para:

$$
\lim _{m \rightarrow \infty} \frac{1}{\sqrt{m}} \cdot \frac{\Gamma\left(\frac{m+1}{2}\right)}{\Gamma\left(\frac{m}{2}\right)}=\frac{1}{\sqrt{2}}
$$

La función gamma

$$
\begin{gathered}
\int_{0}^{\infty} e^{-\mathrm{t}} \cdot t^{\mathrm{m}-1} \cdot \mathrm{d} t=\Gamma(m)=(m-1) \Gamma(m-1)=(m-1) ! \\
(m=1,2,3 \ldots) \\
\Gamma(m+1)=m \cdot \Gamma(\mathrm{m}) ; \Gamma\left(\frac{1}{2}\right)=\sqrt{\pi}
\end{gathered}
$$

Por tanto, si el número de muestras tiende al infinito, resulta:

$$
\lim _{m \rightarrow \infty} g(t)=\frac{1}{\sqrt{2 \pi}} \cdot e^{-t 2 / 2},
$$

y esta es la función de densidad de la distribución normal (curva de campana de Gauss para $\bar{x}=0$ y $s^{2}=1$ ), si se sustituye $t$ por $u$ :

$$
\left(\frac{1}{\sqrt{2 \pi}} \cdot e^{-u^{2} / 2}\right)
$$

Cuanto menor es el número de muestras $n$ tanto más plana resulta la distribución Student y tanto mayor es la dispersión.

Por tanto si se trata de un reducido número de muestras (menos de 30 unidades), en lugar del coeficiente de distribución $u$ hay que usar el coeficiente de distribución $t$; hay que tener en cuenta que el coeficiente de distribución $t$ también depende del número de muestras (tabla 8 y figura 2); por tanto, la fórmula de la figura 1 pasa a $x_{\mathrm{p}}=\bar{x}-t \cdot s$.

En obras cuyo hormigonado se realiza durante un largo período de tiempo puede suceder que el enjuiciamiento de los resultados, si no se han observado las directrices correspondientes, se efectúe demasiado tarde. La figura 3 presenta como ejemplo una obra ficticia, donde los resultados de la valoración del hormigón en masa en función del tiempo han dado valores muy variados. La resistencia mínima requerida $x_{\mathrm{p}}$ alcanzó allí 300 $\mathrm{kp} / \mathrm{cm}^{2}$ para la probabilidad exigida de $F=10 \%$. En la parte superior de la figura se ha registrado el número de muestras no resistentes, y se ve que su frecuencia desciende considerablemente en el transcurso del tiempo. En el primer mes son 14 muestras, en el segundo 6 , en el tercero 2 y en el cuarto solamente una muestra, que no ha alcanzado el valor mínimo $x_{\mathrm{p}}$. Evidentemente la causa de esto fueron las llamadas "dificultades iniciales", a las que se deben muchas veces los daños correspondientes. En forma más marcada se expresan en la parte inferior de la imagen las diferencias que han de atribuirse a esto, donde figuran los resultados procedentes de cada uno de los meses y a todo lo largo del período de la serie según las curvas de campana. La calidad del hormigón, juzgada según la probabilidad o por el número proporcional de muestras, que no han llegado a la resistencia mínima requerida $x_{\mathrm{p}}$ en el período de tiempo considerado, alcanza según la tabla 9: 

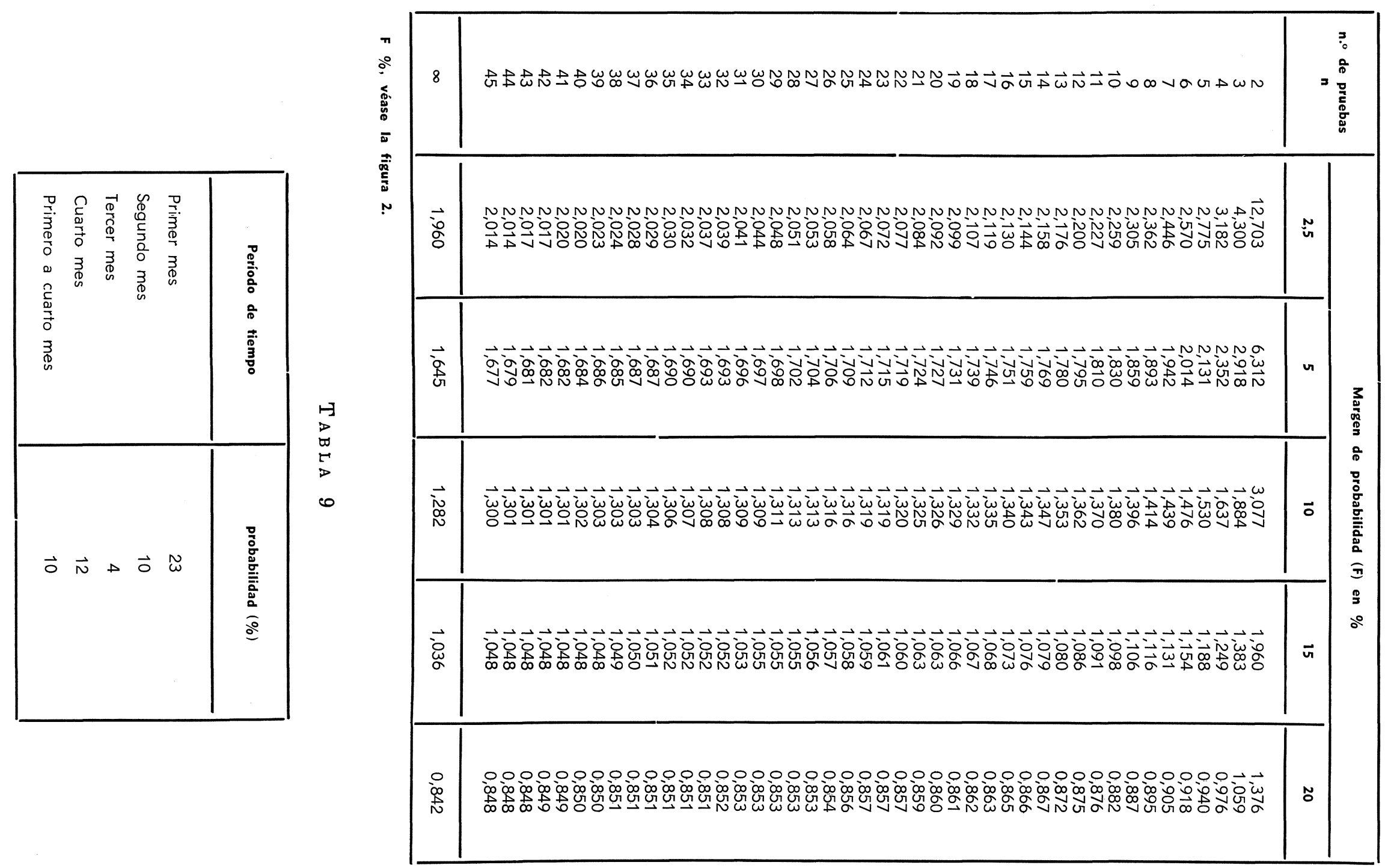

$\infty$ 
En el primer mes puede el hormigón no cumplir, con mucho, el requisito que se le impuso. El coeficiente de seguridad puede resultar en la obra menor que lo prescrito. La importancia de una muestra que no ha cumplido, solamente se valorará si se piensa que una única muestra que no cumple la prueba, de acuerdo con el rendimiento diario, puede representar en algunas circunstancias más de $1.000 \mathrm{~m}^{3}$ de hormigón. Por consiguiente los resultados aumentan en el ejemplo escogido de un mes para otro en razón de su calidad, rápidamente (figura 3), y un enjuiciamiento efectuado a lo largo de todo el período de tiempo de cuatro meses podría suscitar la impresión de que el hormigón estaba en perfectas condiciones y con la misma calidad en todas sus partes.

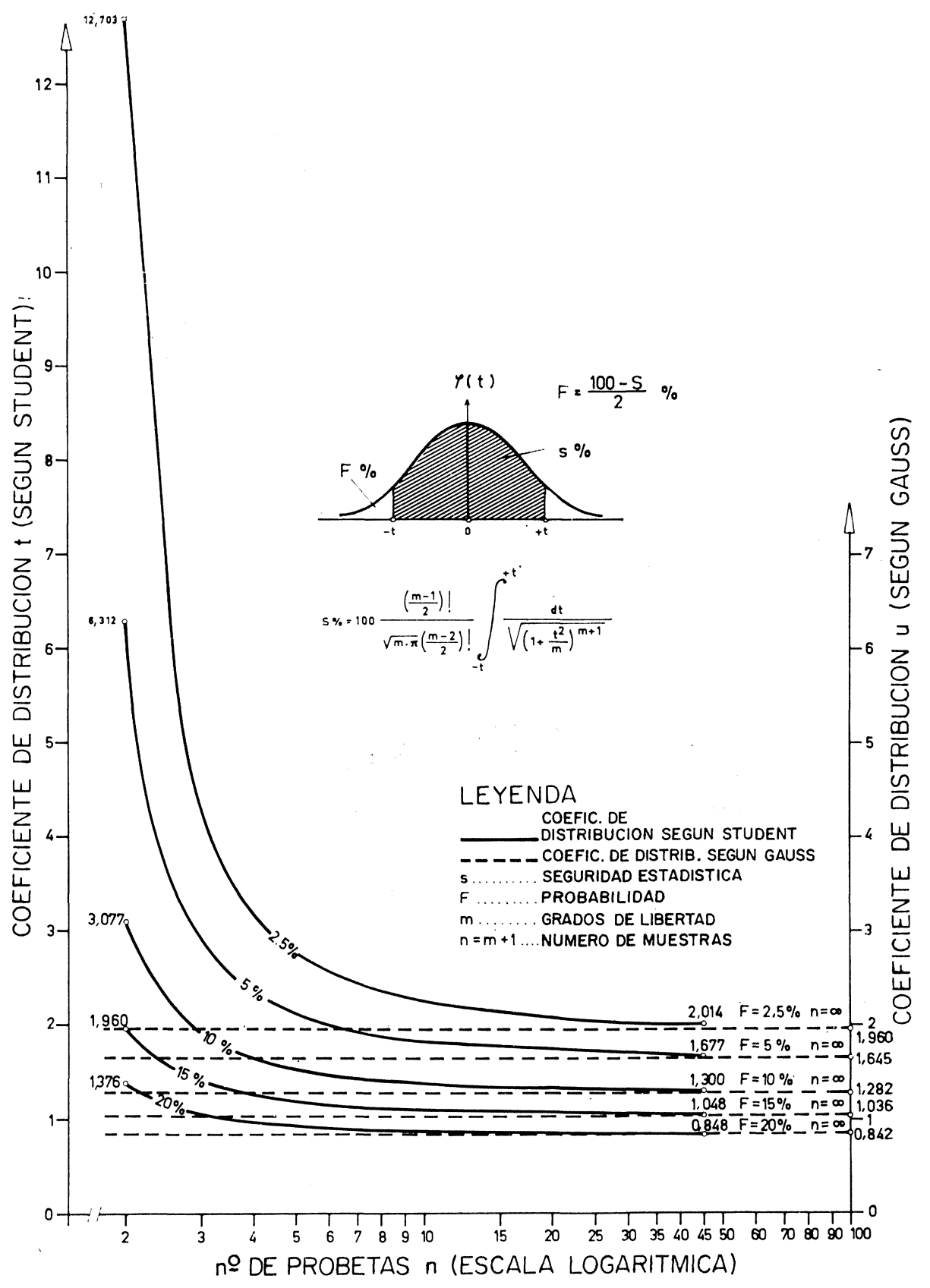

Fig. 2.-Coeficientes de distribución según Student y Gauss en función del número de muestras y del margen de probabilidad. 

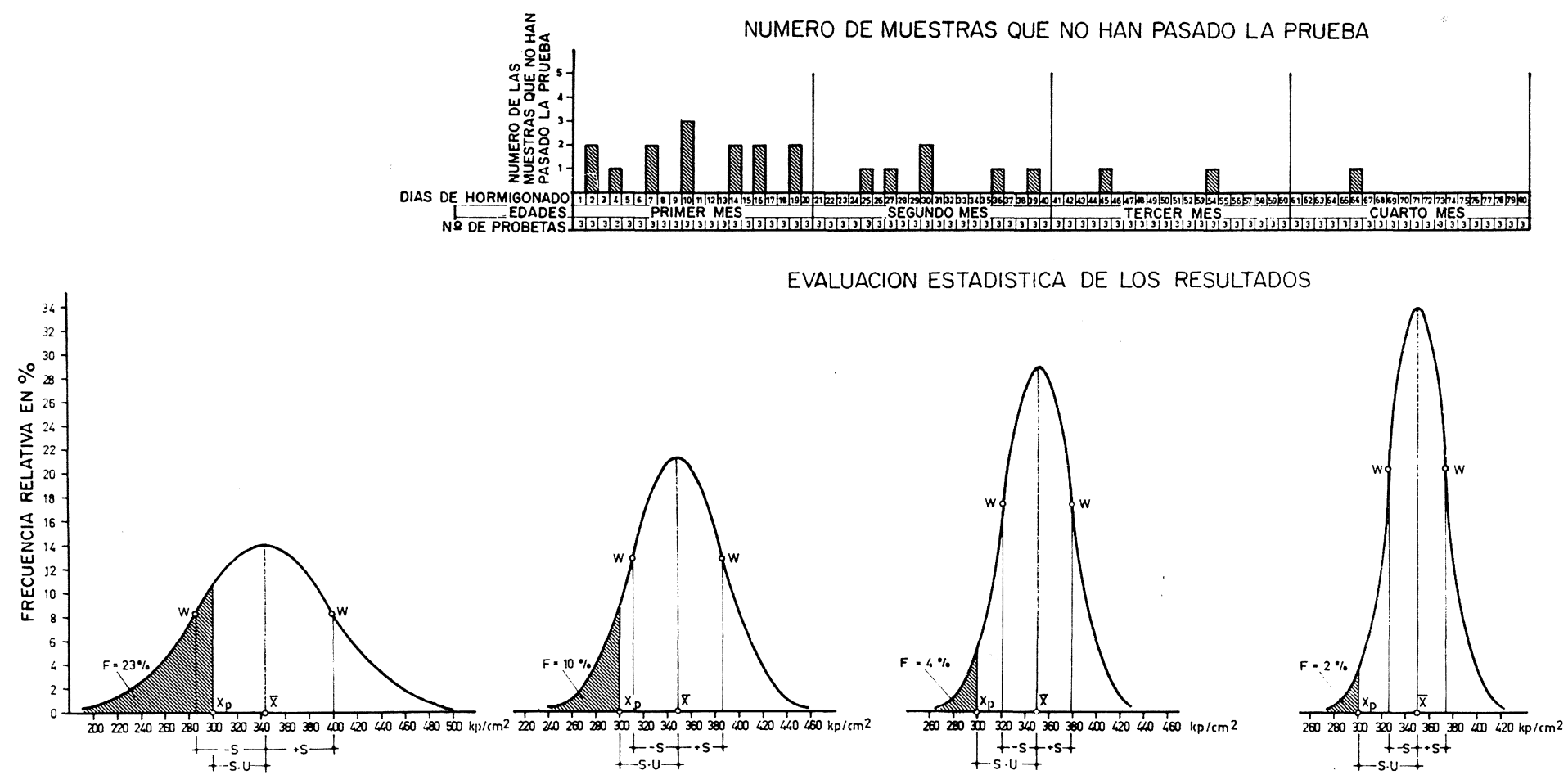

\section{PRIMER MES}

Valor medio aritmético: $X=343 \mathrm{kp} / \mathrm{cm}^{2}$ $s=56.7 \mathrm{kp} / \mathrm{cm}^{2}$.

Coeficiente de variación: $v=16,5 \%$ $F=23 \%$
Número de probetas : $n=60$ unidades Numero de probetas que n han pasado la prueba:

Resistencia mín. requerida : $X_{\mathrm{p}}=300 \mathrm{kp} / \mathrm{cm}^{2}$ Unidad de clase

$20 \mathrm{kp} / \mathrm{cm}^{2}$

SECUNDO MES

Valor medio aritmético:

$X=349 \mathrm{kp} / \mathrm{cm}^{2}$
Desviación estándar

$s=37,5 \mathrm{kp} / \mathrm{cm}^{2}$

variación

Probabilidad:

$F=10 \%$

Número de probetas :

$n=60$ unidades

unero de probetas que no

han pasado la prueba

Resistencia mín. requerida:

$X_{p}=300 \mathrm{kp} / \mathrm{cm}^{2}$

Unidad de clas
$20 \mathrm{kp} / \mathrm{cm}^{2}$

$20 \mathrm{kp} / \mathrm{cm}^{2}$

\section{TERCER MES}

Valor medio aritmético :

$X=350 \mathrm{kp} / \mathrm{cm}^{2}$
Desviación estándar:

$\underset{s=27,8 \mathrm{kp} / \mathrm{cm}^{2}}{\text { Desviación }}$

Coeficiente de variación:

Probabilidad

$F=4 \%$
Número de probetas:

$n=60$ unidades

Número de probetas que no

han pasado la prueba:

Resistencia mín. requerida

$X_{\mathrm{p}}=300 \mathrm{kp} / \mathrm{cm}$

Unidad de clase :
$20 \mathrm{kp} / \mathrm{cm}^{2}$

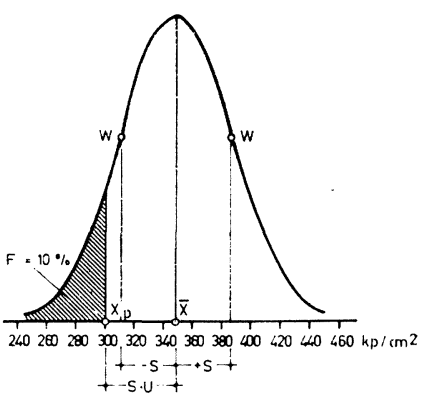

1.0 al 4. MES

Valor medio aritmético:

$X=348 \mathrm{kp} / \mathrm{cm}^{2}$

Coeficiente de variación

$v=11,0 \%$.

$F=10 \%$
Número de probetas:

$n=240$ unidades

Numero de probetas que no

23 unidades $(\approx 10 \%)$

Resistencia min. requerida:

$X_{\mathrm{p}}=300 \mathrm{kp} / \mathrm{cm}^{2}$

Unidad de clase

margen concedido: $F=10 \%$. 
Ahora bien, no resulta dificil comprender que para enjuiciar estadísticamente los resultados de ensayos, el tiempo, en el que transcurre la acción, debe resaltarse siempre con especial cuidado.

Las "dificultades iniciales" mencionadas pueden darse ciertamente en un volumen determinado en cada una de las obras; se deben tener en cuenta convenientemente, y lo mejor es mediante un aumento temporalmente limitado de la medida de predicción $(u \cdot s)$, lo que prácticamente equivale a un aumento de la dosificación de cemento. Pero tan pronto como se haya comprobado que se ha iniciado el servicio en la obra, debe disminuir progresivamente el aumento.

En caso de que aparezcan resultados desfavorables no se puede invocar la conocida propiedad del hormigón, de poder salvar las debilidades existentes en algunos puntos, pues esta característica suya, digna de tenerse en cuenta, se ha considerado ya en la probabilidad previamente establecida, que expresa cuales resultados del ensayo deben quedar por debajo del valor mínimo $x_{\mathrm{p}}$ requerido en la distribución normal supuesta.

Para evitar confusiones se propone la siguiente regla general, que es inequívoca y corresponde al sentido de la estadística: "En una serie de muestras sucesivas, cuyo número de elementos depende de la probabilidad exigida, el valor mínimo $x_{\mathrm{p}}$ sólo dejará de alcanzarse en una única muestra".

Un ejemplo de esta proposición (tabla 10).

Según esto, por ejemplo, con una probabilidad del $5 \%$ de 20 muestras sucesivas sólo una muestra quedará por debajo del valor crítico y con una probabilidad del $15 \%$ en 6 y 7 muestras alternativas sólo se podrá dar una deficiencia.

Debido a las "dificultades iniciales" que se presentan casi regularmente, como se ha mencionado ya, es aconsejable el situar al principio de cada hormigonado la medida de predicción $(u \cdot s)$ superior durante cierto tiempo. La cantidad de este aumento depende de cómo aprecia sus posibilidades el director de obras responsable. Si durante la prueba precedente, adecuada a la importancia y al volumen de la obra, ha conocido suficientemente y ha considerado como buenas la instalación y el proceso de hormigonado así como las capacidades de sus colaboradores y auxiliares, seguramente no le fallará la elección. Un cierto aumento pasajero de la dosificación de cemento de unos 2 a $3 \%$ será conveniente en todo caso. Si, por el contrario, el funcionamiento de la prueba no hubiera eliminado totalmente todas las dificultades, el director de obras prudente situará todavía más elevada, de momento, la medida de predicción.

TABLA 10

\begin{tabular}{|c|c|}
\hline márgen de probabilidad \% & $\begin{array}{c}\text { Número de probetas de la serie que } \\
\text { se deberán tomar para que sólo una } \\
\text { no alcance la resistencia deseada }\end{array}$ \\
\hline 5 & 20 \\
10 & 10 \\
15 & 6 a 7 \\
20 & 5 \\
\hline
\end{tabular}

La figura 4 ilustra un ejemplo de cómo con una resistencia mínima dada $x_{\mathrm{p}}$ el valor medio $\bar{x}$ puede elevarse mediante aumento de la apreciada dispersión $s$ con probabilidad constante y con el coeficiente de distribución $u$ que depende de éste teniendo en cuenta las circunstancias, según la fórmula $\bar{x}=x_{\mathrm{p}}+s \cdot u$, donde $s \cdot u$ signifíca la "medida de predic- 
ción". Las dispersiones $s$ que se ven en la figura van de 15 hasta $50 \mathrm{kp} / \mathrm{cm}^{2}$; abarcan, por tanto, un amplio sector en cuyo centro pudieron encontrarse las desviaciones de los hormigones de nuestras grandes obras perfectamente equipadas y bien conducidas.

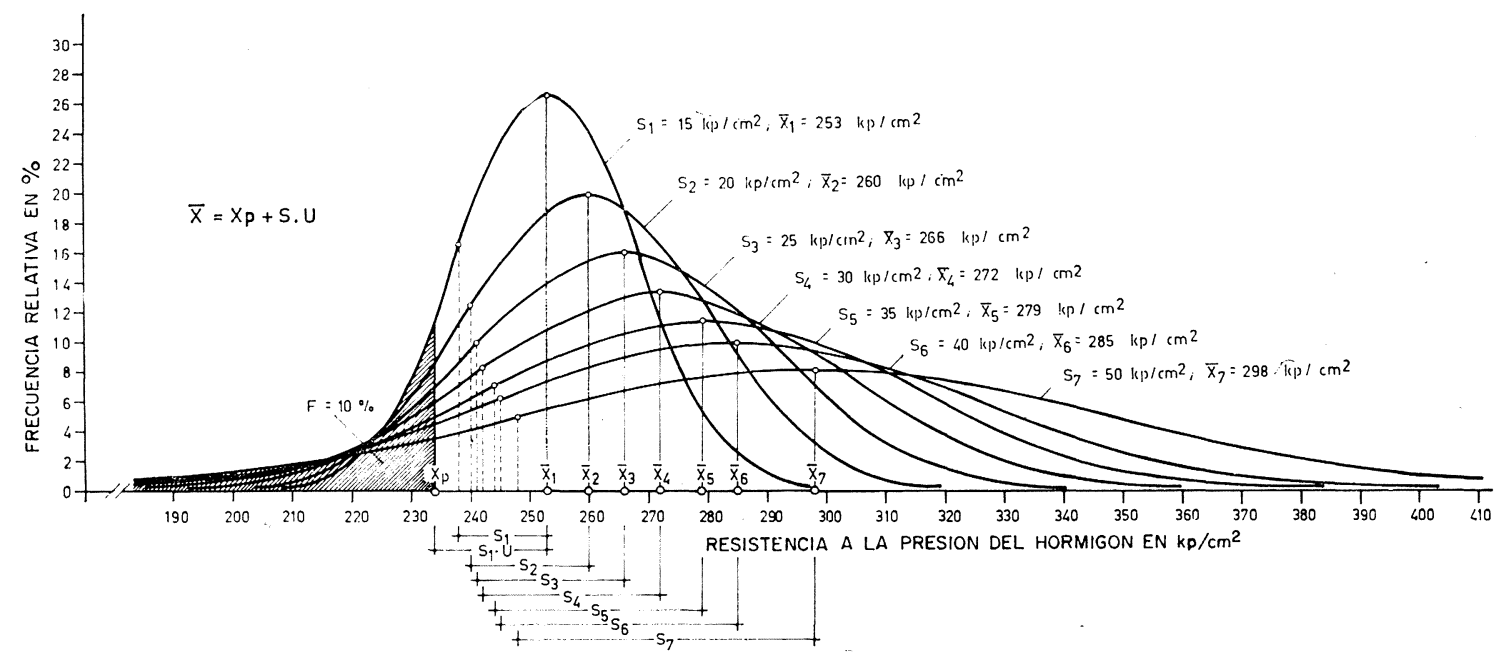

Fig. 4.- Influencia de la desviación estándar $S$ sobre la media de resistencia $X$ con constante $x_{p}=234 \mathrm{kp} / \mathrm{cm}^{2}$ y probabilidad constante $F$.

La mayor parte de las veces, en una obra, que cuenta para el servicio corriente con una dispersión estándar de $30 \mathrm{kp} / \mathrm{cm}^{2}$, bastará aumentar durante un período corto de tiempo la medida de predicción $u \cdot s$ de acuerdo con una desviación $s=40 \mathrm{kp} / \mathrm{cm}^{2}$; sólo en obras dirigidas menos perfectamente, o donde predominan circunstancias dudosas, el director de obras iniciará el hormigonado con $s=50 \mathrm{kp} / \mathrm{cm}^{2}$.

Estas consideraciones valen para un largo período de transición con un gran número de muestras de acuerdo con la distribución normal de Gauss. Por razones económicas, sin embargo, se tenderá a concebir este tiempo muy breve y, por tanto, a tender a contentarse con el número menor posible de muestras. Para este caso se ofrece la distribución Student; sólo que en lugar del coeficiente de distribución $u$ debe emplearse el coeficiente de distribución $t$ (tabla 8 y figura 2).

Al comienzo se indicó ya que en algunas clases de hormigón las resistencias sólo se ensayaron con el tiempo de muestra ya transcurrido. En especial esto se aplica a hormigones en masa con cementos que fraguan lentamente. Tratándose de grandes embalses o presas, la mayor parte de las veces las resistencias de 180 días son decisivas para el enjuiciamiento de la calidad del hormigón.

Pero puede suceder fácilmente que algunas muestras aisladas de 180 días no cumplan las condiciones de resistencia, aún cuando los resultados averiguados por precaución en fechas anteriores permiten esperar resistencias posteriores perfectas. En un caso tan extraordinario el perito en estadísticas, responsable oficialmente, decidirá sobre la aceptación o denegación del hormigón correspondiente a estas muestras defectuosas; pues él es el encargado, entre otras cosas, de la vigilancia del factor de seguridad $\nu$ y valiéndose de las isobaras de la obra en el lugar incriminado puede comprobar el valor $\nu$ real y, además, también averiguar si no se puede reducir algo, para esta parte especialmente restringida de la obra, la correspondiente distancia de seguridad $\sigma_{\max }(\nu-1)$ (figura 1), comprimiéndose la requerida resistencia mínima $x_{\mathrm{p}}$, posiblemente en la medida en que teniendo en cuenta la correspondiente seguridad residual se cumple finalmente la probabilidad. 
El estadístico tomará fácilmente la decisión, especialmente en los casos en que, en todo el campo de una calidad de hormigón, se diferencian notablemente las máximas tensiones de la obra $\sigma_{\text {max }}$ de un lugar a otro.

Del hecho de que en los embalses o presas en general únicamente sólo pueda medirse la calidad del hormigón al cabo de medio año, es decir, en un momento en el que la obra, si no ya construida, puede haber avanzado ya considerablemente, se deriva la obligación de aplicar un cuidado extraordinario en la fabricación del hormigón especialmente al comienzo de los trabajos.

\section{B I B L I O G R A F I A}

(1) Tremmel, E. und Wogrin, A.: Mathematisch-statistische Auswertung der Güteprüfungen von Massenbeton. Der Bauingenieur (1955) Heft 1. S.' 28-32. Springer-Verlag Berlin-Göttingen-Heildelberg.

(2) Bonzel, E. und Dahms J.: Über die Bedeutung der statistischen Qualitätskontrolle bei Beton. beton 10 (1964), S. 429-436.

(3) Rüsch, H.: Zur statistischen Qualitätskontrolle des Betons Materialprüfung 6 (1964), Nr. 11, S. 387-394.

(4) ACI Standard 214-65: Recomended practice for evaluation of compression test results of field concrete.

(5) Graf, Henning, Stange.: Formeln und Tabellen der mathematischen Statistik. Springer-Verlag. 1966.

(6) KREYszIG, E.: Statistische Methoden und ihre Anwendungen. Vandenhoeck und Rupprecht, Göttingen, 1970.

(7) Heinhold-Gaede.: Ingenieur-Statistik. R. Oldenburg München-Wien, 1968 